\title{
Shared Depletion and Re-stabilization Colloidal Interactions in Phase Diagrams for Silica Nanoparticle and Asphaltene + Polystyrene + Solvent Mixtures
}

\author{
Anupam Kumar, Sourav Chowdhury, John M. Shaw*
}

Department of Chemical and Materials Engineering, University of Alberta, Edmonton, Alberta, T6G 2V4 Canada

\section{Corresponding Author}

*John M. Shaw (jmshaw@ualberta.ca)

\section{Supporting Information}

Experimental data sets for relative phase volumes, phase boundary enclosing gas $(\mathrm{G})$ and liquid (L) phases at equilibrium and critical points for nano-silica + polystyrene $(237 \mathrm{~kg} / \mathrm{mol})+$ cyclohexane are reported at $296 \mathrm{~K}, 303 \mathrm{~K}$, and $313 \mathrm{~K}$ as Tables S1a-c, Table S2a-c and Table S3a-c respectively.

Cloud points data for polystyrene + cyclohexane, polystyrene + cyclohexane + toluene, polystyrene + cyclohexane + heptane, and polystyrene + cyclohexane + water are reported in Tables S4, S5, S6, and S7 respectively.

Compositions and associated polymer-rich phase volume data for Athabasca retentate + polystyrene $(400 \mathrm{~kg} / \mathrm{mol})+$ toluene (based on X-ray measurements) for trajectories $\mathrm{p}$, $\mathrm{q}$, and $\mathrm{r}$ are reported in Table S8. Computed binodal points for this ternary, based on X-ray measurements, are reported in Table S9. 
Table S.10: Compositions and associated polymer-rich phase volume data for pentane asphaltene + polystyrene + toluene (based on X-ray measurements) for trajectories $1,2,3,4,5,6,7$

Compositions and associated polymer-rich phase volume data for pentane asphaltene + polystyrene + toluene for trajectories $\mathrm{p}, \mathrm{q}$, and $\mathrm{r}$ (based on X-ray measurements) and computed binodal points, using the method of Bondar et al..$^{40}$ are listed in Table S 11 and S 13 respectively.

Table S 12: Compositions and associated polymer-rich phase volume data for retentate + polystyrene + toluene (based on X-Ray measurements) for seven composition trajectories. 


\begin{tabular}{|c|c|c|c|}
\hline Table S & a Polymer-Ri & Phase Volume Frac & n Data at $296 \mathrm{~K}$ \\
\hline $\begin{array}{l}\text { Polystyre } \\
\text { ne mass } \\
\text { fraction } \\
\pm \quad 0.0005 \\
\end{array}$ & $\begin{array}{c}\text { Nano silica } \\
\text { mass } \\
\text { fraction } \\
\pm 0.0005 \\
\end{array}$ & $\begin{array}{c}\text { Cyclohexane mass } \\
\text { fraction } \\
\pm 0.0005\end{array}$ & $\begin{array}{l}\text { volume fraction } \\
\text { of polymer rich } \\
\text { phase }\left(R_{1}\right) \pm 0.03\end{array}$ \\
\hline 0.0002 & 0.0000 & 0.9998 & 1.0000 \\
\hline 0.0002 & 0.0013 & 0.9986 & 0.8500 \\
\hline 0.0002 & 0.0028 & 0.9970 & 0.5500 \\
\hline 0.0002 & 0.0044 & 0.9954 & 0.0000 \\
\hline 0.0039 & 0.0000 & 0.9961 & 1.0000 \\
\hline 0.0039 & 0.0033 & 0.9927 & 0.6800 \\
\hline 0.0039 & 0.0061 & 0.9900 & 0.1100 \\
\hline 0.0039 & 0.0066 & 0.9895 & 0.0000 \\
\hline 0.0105 & 0.0000 & 0.9895 & 1.0000 \\
\hline 0.0104 & 0.0011 & 0.9885 & 0.8900 \\
\hline 0.0101 & 0.0031 & 0.9868 & 0.7300 \\
\hline 0.0101 & 0.0056 & 0.9843 & 0.5700 \\
\hline 0.0101 & 0.0095 & 0.9805 & 0.2100 \\
\hline 0.0100 & 0.0110 & 0.9790 & 0.0400 \\
\hline 0.0095 & 0.0000 & 0.9905 & 1.0000 \\
\hline 0.0095 & 0.0008 & 0.9897 & 0.8200 \\
\hline 0.0095 & 0.0033 & 0.9872 & 0.5700 \\
\hline 0.0094 & 0.0081 & 0.9825 & 0.0000 \\
\hline 0.0500 & 0.0000 & 0.9500 & 0.8800 \\
\hline 0.0515 & 0.0003 & 0.9483 & 0.8700 \\
\hline 0.0512 & 0.0051 & 0.9437 & 0.7800 \\
\hline 0.0507 & 0.0131 & 0.9362 & 0.5100 \\
\hline 0.0506 & 0.0168 & 0.9326 & 0.3500 \\
\hline 0.0504 & 0.0215 & 0.9281 & 0.2600 \\
\hline
\end{tabular}




\begin{tabular}{|c|c|c|c|}
\hline 0.0502 & 0.0249 & 0.9249 & 0.1400 \\
\hline 0.0500 & 0.0278 & 0.9222 & 0.0750 \\
\hline 0.0500 & 0.0300 & 0.9200 & 0.0000 \\
\hline 0.1000 & 0.0000 & 0.9000 & 0.6700 \\
\hline 0.1000 & 0.0100 & 0.8900 & 0.6710 \\
\hline 0.0980 & 0.0145 & 0.8875 & 0.6200 \\
\hline 0.0960 & 0.0250 & 0.8790 & 0.5300 \\
\hline 0.0970 & 0.0290 & 0.8740 & 0.3600 \\
\hline 0.0960 & 0.0360 & 0.8680 & 0.1200 \\
\hline 0.0970 & 0.0430 & 0.8600 & 0.0760 \\
\hline 0.0950 & 0.0530 & 0.8520 & 0.0310 \\
\hline 0.1500 & 0.0000 & 0.8500 & 0.3500 \\
\hline 0.1463 & 0.0013 & 0.8524 & 0.3400 \\
\hline 0.1461 & 0.0029 & 0.8510 & 0.3200 \\
\hline 0.1452 & 0.0069 & 0.8479 & 0.3300 \\
\hline 0.1440 & 0.0176 & 0.8384 & 0.2660 \\
\hline 0.1677 & 0.0220 & 0.8103 & 0.2400 \\
\hline 0.1427 & 0.0263 & 0.8310 & 0.1300 \\
\hline 0.1410 & 0.0377 & 0.8213 & 0.0000 \\
\hline 0.2121 & 0.0000 & 0.7879 & 0.0730 \\
\hline 0.2100 & 0.0006 & 0.7894 & 0.0700 \\
\hline 0.2116 & 0.0024 & 0.7860 & 0.0580 \\
\hline 0.2107 & 0.0068 & 0.7825 & 0.0800 \\
\hline 0.2099 & 0.0109 & 0.7792 & 0.0820 \\
\hline 0.2088 & 0.0161 & 0.7752 & 0.0400 \\
\hline 0.2079 & 0.0201 & 0.7720 & 0.0380 \\
\hline 0.2066 & 0.0260 & 0.7674 & 0.0220 \\
\hline
\end{tabular}


Table S1b LG/G and LG/L Phase Boundary Data at 296 K

\begin{tabular}{|c|c|c|}
\hline $\begin{array}{c}\text { Nano silica mass fraction } \\
\pm 0.0005\end{array}$ & $\begin{array}{c}\text { Polystyrene mass fraction } \\
\pm 0.0005\end{array}$ & $\begin{array}{c}\text { Cyclohexane mass fraction } \\
\pm 0.0005\end{array}$ \\
\hline 0.0044 & 0.0016 & 0.9940 \\
\hline 0.0066 & 0.0039 & 0.9895 \\
\hline 0.0110 & 0.0100 & 0.9790 \\
\hline 0.0300 & 0.0500 & 0.9200 \\
\hline 0.0530 & 0.0950 & 0.8520 \\
\hline 0.0377 & 0.1410 & 0.8213 \\
\hline 0.0260 & 0.2066 & 0.7674 \\
\hline 0.0000 & 0.2300 & 0.7700 \\
\hline 0.0000 & 0.0200 & 0.9800 \\
\hline 0.0002 & 0.0001 & 0.9997 \\
\hline 0.0002 & 0.0000 & 0.9998 \\
\hline 0.0043 & 0.0001 & 0.9956 \\
\hline
\end{tabular}

\begin{tabular}{|c|c|c|}
\hline \multicolumn{2}{|c|}{ Table S1c L=G Critical Point at 296 $\mathrm{K}$} \\
\hline $\begin{array}{c}\text { Nano silica mass } \\
\text { fraction }\end{array}$ & $\begin{array}{c}\text { Polystyrene mass } \\
\text { fraction }\end{array}$ & $\begin{array}{c}\text { Cyclohexane mass } \\
\text { fraction }\end{array}$ \\
\hline $0.0030 \pm 0.00005$ & $0.0002 \pm 0.00005$ & $0.9968 \pm 0.00005$ \\
\hline
\end{tabular}

\begin{tabular}{|c|c|c|c|}
\hline $\begin{array}{c}\text { Nano silica } \\
\text { mass } \\
\text { fraction } \\
\pm 0.0005\end{array}$ & $\begin{array}{c}\text { Polystyrene } \\
\text { mass } \\
\text { fraction } \\
\pm 0.0005\end{array}$ & $\begin{array}{c}\text { Cyclohexane mass } \\
\text { fraction } \\
\pm 0.0005\end{array}$ & $\begin{array}{l}\text { volume fraction } \\
\text { of polymer rich } \\
\text { phase }\left(R_{1}\right) \pm 0.03\end{array}$ \\
\hline 0.0000 & 0.0002 & 0.9998 & 1.0000 \\
\hline 0.0013 & 0.0002 & 0.9986 & 0.8400 \\
\hline 0.0028 & 0.0002 & 0.9970 & 0.5800 \\
\hline 0.0044 & 0.0016 & 0.9940 & 0.0000 \\
\hline
\end{tabular}




\begin{tabular}{|c|c|c|c|}
\hline 0.0000 & 0.0039 & 0.9961 & 1.0000 \\
\hline 0.0033 & 0.0039 & 0.9927 & 0.7000 \\
\hline 0.0061 & 0.0039 & 0.9900 & 0.1200 \\
\hline 0.0066 & 0.0039 & 0.9895 & 0.0000 \\
\hline 0.0000 & 0.0105 & 0.9895 & 1.0000 \\
\hline 0.0011 & 0.0104 & 0.9885 & 0.9200 \\
\hline 0.0031 & 0.0101 & 0.9868 & 0.7800 \\
\hline 0.0056 & 0.0101 & 0.9843 & 0.4980 \\
\hline 0.0095 & 0.0101 & 0.9805 & 0.2500 \\
\hline 0.0110 & 0.0100 & 0.9790 & 0.0300 \\
\hline 0.0000 & 0.0095 & 0.9905 & 1.0000 \\
\hline 0.0008 & 0.0095 & 0.9897 & 0.8500 \\
\hline 0.0033 & 0.0095 & 0.9872 & 0.6300 \\
\hline 0.0081 & 0.0094 & 0.9825 & 0.0000 \\
\hline 0.0000 & 0.0200 & 0.9800 & 1.0000 \\
\hline 0.0010 & 0.0200 & 0.9790 & 0.9900 \\
\hline 0.0034 & 0.0200 & 0.9767 & 0.7300 \\
\hline 0.0128 & 0.0198 & 0.9674 & 0.1500 \\
\hline 0.0143 & 0.0197 & 0.9660 & 0.1000 \\
\hline 0.0172 & 0.0197 & 0.9631 & 0.0000 \\
\hline 0.0000 & 0.0500 & 0.9500 & 1.0000 \\
\hline 0.0003 & 0.0515 & 0.9483 & 1.0000 \\
\hline 0.0051 & 0.0512 & 0.9437 & 0.7700 \\
\hline 0.0131 & 0.0507 & 0.9362 & 0.3600 \\
\hline 0.0168 & 0.0506 & 0.9326 & 0.2700 \\
\hline 0.0215 & 0.0504 & 0.9281 & 0.2300 \\
\hline 0.0249 & 0.0502 & 0.9249 & 0.1300 \\
\hline 0.0278 & 0.0500 & 0.9222 & 0.0780 \\
\hline
\end{tabular}




\begin{tabular}{|c|c|c|c|}
\hline 0.0300 & 0.0500 & 0.9200 & 0.0000 \\
\hline 0.0000 & 0.1000 & 0.9000 & 1.0000 \\
\hline 0.0100 & 0.1000 & 0.8900 & 0.6800 \\
\hline 0.0145 & 0.0980 & 0.8875 & 0.5500 \\
\hline 0.0250 & 0.0960 & 0.8790 & 0.2400 \\
\hline 0.0290 & 0.0970 & 0.8740 & 0.0400 \\
\hline 0.0360 & 0.0960 & 0.8680 & 0.0000 \\
\hline 0.0000 & 0.1500 & 0.8500 & 1.0000 \\
\hline 0.0013 & 0.1463 & 0.8524 & 0.9730 \\
\hline 0.0029 & 0.1461 & 0.8510 & 0.7430 \\
\hline 0.0069 & 0.1452 & 0.8479 & 0.6870 \\
\hline 0.0176 & 0.1440 & 0.8384 & 0.5230 \\
\hline 0.0220 & 0.1677 & 0.8103 & 0.3170 \\
\hline 0.0263 & 0.1427 & 0.8310 & 0.0000 \\
\hline 0.0000 & 0.2121 & 0.7879 & 1.0000 \\
\hline 0.0006 & 0.2100 & 0.7894 & 0.9250 \\
\hline 0.0024 & 0.2116 & 0.7860 & 0.7620 \\
\hline 0.0068 & 0.2107 & 0.7825 & 0.4600 \\
\hline 0.0109 & 0.2099 & 0.7792 & 0.2600 \\
\hline 0.0161 & 0.2088 & 0.7752 & 0.0100 \\
\hline 0.0012 & 0.2621 & 0.7367 & 1.0000 \\
\hline 0.0097 & 0.2598 & 0.7305 & 0.2300 \\
\hline 0.0123 & 0.2592 & 0.7285 & 0.0000 \\
\hline
\end{tabular}

\begin{tabular}{|c|c|c|}
\hline \multicolumn{2}{|c|}{ Table S2b LG/G and LG/L Phase Boundary Data at $303 \mathrm{~K}$} \\
\hline $\begin{array}{c}\text { Nano silica mass } \\
\text { fraction }\end{array}$ & $\begin{array}{c}\text { Polystyrene mass } \\
\text { fraction }\end{array}$ & $\begin{array}{c}\text { Cyclohexane mass } \\
\pm 0.0005\end{array}$ \\
\pm 0.0005 & \pm 0.0005 \\
\hline 0.0044 & 0.0016 & 0.9940 \\
\hline 0.0066 & 0.0039 & 0.9895 \\
\hline
\end{tabular}




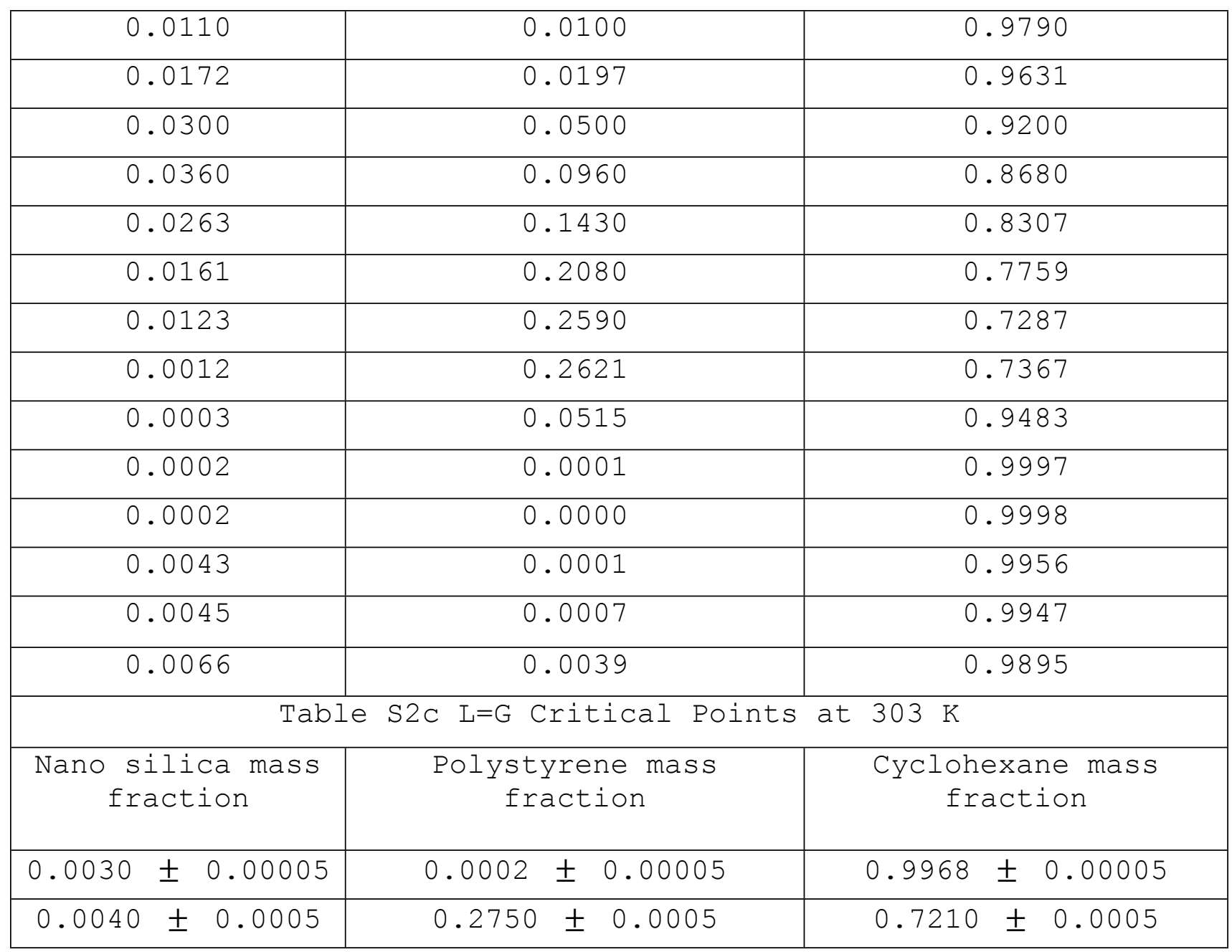

\begin{tabular}{|c|c|c|c|}
\hline \multicolumn{2}{|c|}{ Table S3a } & Polymer-Rich Phase Volume Fraction Data at $313 \mathrm{~K}$ \\
\hline $\begin{array}{c}\text { Nano silica } \\
\text { mass } \\
\begin{array}{c}\text { fraction } \\
\pm 0.0005\end{array}\end{array}$ & $\begin{array}{c}\text { Polystyrene mass } \\
\text { fraction } \\
\pm 0.0005\end{array}$ & $\begin{array}{c}\text { Cyclohexane mass } \\
\text { fraction } \\
\pm 0.0005\end{array}$ & $\begin{array}{c}\text { volume } \\
\text { fraction of } \\
\text { polymer rich } \\
\text { phase }\left(\mathrm{R}_{1}\right) \pm \\
0.03\end{array}$ \\
\hline 0.0021 & 0.0022 & 0.9957 & 0.8740 \\
\hline 0.0046 & 0.0022 & 0.9932 & 0.0000 \\
\hline & & & 0.6811 \\
\hline 0.9860 & 0.0097 & 0.0043 & 0.5011 \\
\hline 0.9813 & 0.0097 & 0.0090 & \\
\hline
\end{tabular}




\begin{tabular}{|l|l|l|l|}
\hline 0.9768 & 0.0096 & 0.0135 & 0.2200 \\
\hline & & & \\
\hline 0.8866 & 0.1069 & 0.0065 & 0.8095 \\
\hline 0.8803 & 0.1061 & 0.0136 & 0.5646 \\
\hline 0.8752 & 0.1055 & 0.0193 & 0.3110 \\
\hline 0.8716 & 0.1051 & 0.0233 & 0.1752 \\
\hline & & & 0.5842 \\
\hline 0.8025 & 0.1899 & 0.0076 & 0.3794 \\
\hline 0.7999 & 0.1893 & 0.0108 & 0.2378 \\
\hline 0.7981 & 0.1889 & 0.0130 & 0.7136 \\
\hline & & & 0.3456 \\
\hline 0.7993 & 0.1939 & 0.0068 & 1.0000 \\
\hline 0.7939 & 0.1926 & 0.0136 & \\
\hline & & & 0.2401 \\
\hline 0.7557 & 0.0042 & & \\
\hline
\end{tabular}

Table S3b LG/G and LG/L Phase Boundary Data at $313 \mathrm{~K}$

Nano silica mass fraction $\pm 0.0005$ 0.0017

0.0046

0.0182

0.0277

0.0167

0.0049

0.0010

0.0014

0.0010
Polystyrene mass fraction $\pm 0.0005$ 0.0022 0.0022 0.0096 0.1046

0.1882

0.2802

0.1912

0.1074

0.0098
Cyclohexane mass fraction $\pm 0.0005$ 0.9961 0.9932 0.9722 0.8677

0.7951

0.7149

0.8078

0.8912

Table S3c L=G Critical Points at $313 \mathrm{~K}$

Nano silica mass fraction Polystyrene mass fraction Cyclohexane mass fraction

$0.0042 \pm 0.0005$
$0.0030 \pm 0.00005$

$0.2601 \pm 0.0005$

$0.7357 \pm 0.0005$

$0.0002 \pm 0.00005$

$0.9968 \pm 0.00005$ 



\begin{tabular}{|c|c|}
\hline \multicolumn{2}{|c|}{ Table S4 Cloud Point Data for Cyclohexane + Polystyrene $(237 \mathrm{~kg} / \mathrm{mol})$ Binary } \\
\hline $\begin{array}{c}\text { Polystyrene mass fraction } \\
\pm 0.005\end{array}$ & $\begin{array}{c}\text { Temperature }(\mathrm{K}) \\
\pm 0.20\end{array}$ \\
\hline 0.01 & 296.56 \\
\hline 0.0267 & 298.46 \\
\hline 0.05 & 298.56 \\
\hline 0.087 & 298.66 \\
\hline 0.1 & 298.56 \\
\hline 0.138 & 298.26 \\
\hline 0.198 & 297.86 \\
\hline
\end{tabular}

\begin{tabular}{|c|c|}
\hline Table S5a Cloud Point Data for Cyclohexane + Polystyrene $(237 \mathrm{~kg} / \mathrm{mol})+$ Toluene $(1$ mass $\%)$ \\
\hline $\begin{array}{c}\text { Polystyrene mass fraction } \\
\pm 0.005\end{array}$ & $\begin{array}{c}\text { Temperature }(\mathrm{K}) \\
\pm 0.20\end{array}$ \\
\hline 0.01 & 294.26 \\
\hline 0.0267 & 296.36 \\
\hline 0.05 & 296.46 \\
\hline 0.1 & 296.66 \\
\hline 0.15 & 296.16 \\
\hline
\end{tabular}

\begin{tabular}{|c|c|}
\hline Table S5b Cloud Point Data for Cyclohexane + Polystyrene $(237 \mathrm{~kg} / \mathrm{mol})+$ Toluene $(5$ mass $\%)$ \\
\hline $\begin{array}{c}\text { Polystyrene mass fraction } \\
\pm 0.005\end{array}$ & $\begin{array}{c}\text { Temperature }(\mathrm{K}) \\
\pm 0.20\end{array}$ \\
0.01 & 287.06 \\
\hline 0.0267 & 289.06 \\
\hline 0.05 & 289.06 \\
\hline 0.1 & 289.26 \\
\hline 0.15 & 288.06 \\
\hline
\end{tabular}




\begin{tabular}{|c|c|}
\hline Table S6a Cloud Point Data for Cyclohexane + Polystyrene (237 kg/mol) + Heptane (4.5 mass \%) \\
\hline $\begin{array}{c}\text { Polystyrene mass fraction } \\
\pm 0.005\end{array}$ & $\begin{array}{c}\text { Temperature }(\mathrm{K}) \\
\pm 0.20\end{array}$ \\
\hline 0.01 & 302.16 \\
\hline 0.05 & 302.7 \\
\hline 0.0874 & 303.96 \\
\hline 0.1987 & 303.26 \\
\hline
\end{tabular}

\begin{tabular}{|c|c|}
\hline Table S6b Cloud Point Data for Cyclohexane + Polystyrene $(237 \mathrm{~kg} / \mathrm{mol})+$ Heptane $(8.3$ mass $\%)$ \\
\hline $\begin{array}{c}\text { Polystyrene mass fraction } \\
\pm 0.005\end{array}$ & $\begin{array}{c}\text { Temperature }(\mathrm{K}) \\
\pm 0.20\end{array}$ \\
\hline 0.01 & 302.16 \\
\hline 0.05 & 302.7 \\
\hline 0.0874 & 303.96 \\
\hline 0.1987 & 303.26 \\
\hline
\end{tabular}

\begin{tabular}{|c|c|}
\hline Table S7 Cloud Point Data for Cyclohexane + Polystyrene $(237 \mathrm{~kg} / \mathrm{mol})+$ Water (saturated) \\
\hline $\begin{array}{c}\text { Polystyrene mass fraction } \\
\pm 0.005\end{array}$ & $\begin{array}{c}\text { Temperature }(\mathrm{K}) \\
\pm 0.20\end{array}$ \\
\hline 0.01 & 297.76 \\
\hline 0.0267 & 299.66 \\
\hline 0.05 & 299.76 \\
\hline 0.0874 & 299.86 \\
\hline 0.1 & 299.76 \\
\hline 0.1384 & 299.46 \\
\hline
\end{tabular}


Table S.8: Compositions and associated polymer-rich phase volume data for Athabasca retentate + polystyrene + toluene (based on X-ray measurements) for trajectories $\mathrm{p}, \mathrm{q}$, and $\mathrm{r}$

\begin{tabular}{|c|c|c|c|c|c|}
\hline \multirow{7}{*}{$\mathrm{p}$} & $\begin{array}{l}\text { Asphaltene } \\
\text { volume } \\
\text { fraction }\end{array}$ & $\begin{array}{l}\text { Asphaltene } \\
\text { mass } \\
\text { fraction }\end{array}$ & $\begin{array}{l}\text { Polystyrene } \\
\text { volume } \\
\text { fraction }\end{array}$ & $\begin{array}{l}\text { Polystyrene } \\
\text { mass } \\
\text { fraction }\end{array}$ & $\begin{array}{l}\text { Volume } \\
\text { fraction of the } \\
\text { upper phase } \\
\left(\mathrm{R}_{1}\right)\end{array}$ \\
\hline & 0.053 & 0.069 & 0.071 & 0.083 & 0.760 \\
\hline & 0.048 & 0.063 & 0.064 & 0.076 & 0.775 \\
\hline & 0.044 & 0.058 & 0.059 & 0.070 & 0.798 \\
\hline & 0.039 & 0.052 & 0.052 & 0.062 & 0.829 \\
\hline & 0.034 & 0.045 & 0.045 & 0.054 & 0.880 \\
\hline & 0.030 & 0.040 & 0.040 & 0.048 & 0.911 \\
\hline \multirow{6}{*}{$q$} & 0.100 & 0.128 & 0.043 & 0.050 & 0.371 \\
\hline & 0.093 & 0.120 & 0.040 & 0.046 & 0.393 \\
\hline & 0.085 & 0.110 & 0.036 & 0.042 & 0.427 \\
\hline & 0.075 & 0.098 & 0.032 & 0.037 & 0.484 \\
\hline & 0.070 & 0.092 & 0.030 & 0.035 & 0.517 \\
\hline & 0.063 & 0.083 & 0.027 & 0.032 & 0.576 \\
\hline \multirow{5}{*}{$\mathrm{r}$} & 0.13 & 0.164 & 0.03 & 0.034 & 0.13 \\
\hline & 0.12 & 0.153 & 0.02 & 0.023 & 0.16 \\
\hline & 0.10 & 0.129 & 0.02 & 0.023 & 0.19 \\
\hline & 0.09 & 0.117 & 0.02 & 0.023 & 0.24 \\
\hline & 0.08 & 0.104 & 0.02 & 0.023 & 0.29 \\
\hline
\end{tabular}


Table S.9: Computed binodal points for Athabasca retentate + polystyrene + toluene based on Xray measurements for composition trajectories p, q, r in Table S.8 and the method of Bondar et al. ${ }^{40}$

\begin{tabular}{|c|c|}
\hline Asphaltene volume fraction & Polystyrene volume fraction \\
\hline 0.020 & 0.066 \\
\hline 0.021 & 0.050 \\
\hline 0.021 & 0.058 \\
\hline 0.021 & 0.074 \\
\hline 0.022 & 0.082 \\
\hline 0.023 & 0.042 \\
\hline 0.118 & 0.011 \\
\hline 0.125 & 0.013 \\
\hline 0.129 & 0.012 \\
\hline 0.135 & 0.013 \\
\hline 0.139 & 0.013 \\
\hline 0.142 & 0.013 \\
\hline
\end{tabular}


Table S.10: Compositions and associated polymer-rich phase volume data for pentane asphaltene + polystyrene + toluene (based on X-ray measurements) for trajectories $1,2,3,4,5,6,7$

\begin{tabular}{|c|c|c|c|c|}
\hline $\begin{array}{c}\text { Asphaltene } \\
\text { volume } \\
\text { fraction }\end{array}$ & $\begin{array}{c}\text { Asphaltene mass } \\
\text { fraction }\end{array}$ & $\begin{array}{c}\text { polymer volume } \\
\text { fraction }\end{array}$ & $\begin{array}{c}\text { Polymer mass } \\
\text { fraction }\end{array}$ & $\begin{array}{c}\mathrm{R}_{1} \text { (upper phase } \\
\text { volume fraction) }\end{array}$ \\
\hline 0.040 & 0.054 & 0.034 & 0.041 & 0.900 \\
\hline 0.049 & 0.066 & 0.033 & 0.039 & 0.820 \\
\hline 0.076 & 0.101 & 0.032 & 0.038 & 0.650 \\
\hline 0.100 & 0.131 & 0.032 & 0.037 & 0.500 \\
\hline 0.112 & 0.147 & 0.031 & 0.036 & 0.440 \\
\hline 0.148 & 0.191 & 0.030 & 0.034 & 0.310 \\
\hline 0.170 & 0.218 & 0.029 & 0.033 & 0.170 \\
\hline 0.185 & 0.236 & 0.029 & 0.033 & 0.100 \\
\hline
\end{tabular}

\begin{tabular}{|c|c|c|c|c|}
\hline \multicolumn{2}{|c|}{2} \\
$\begin{array}{c}\text { Asphaltene } \\
\text { volume } \\
\text { fraction }\end{array}$ & $\begin{array}{c}\text { Asphaltene mass } \\
\text { fraction }\end{array}$ & $\begin{array}{c}\text { Polymer volume } \\
\text { fraction }\end{array}$ & $\begin{array}{c}\text { Polymer mass } \\
\text { fraction }\end{array}$ & $\begin{array}{c}\mathrm{R}_{1} \text { (upper phase } \\
\text { volume fraction) }\end{array}$ \\
\hline 0.048 & 0.064 & 0.067 & 0.079 & 0.860 \\
\hline 0.066 & 0.087 & 0.065 & 0.076 & 0.700 \\
\hline 0.091 & 0.119 & 0.064 & 0.075 & 0.590 \\
\hline 0.130 & 0.168 & 0.061 & 0.070 & 0.490 \\
\hline 0.190 & 0.241 & 0.057 & 0.064 & 0.320 \\
\hline 0.243 & 0.303 & 0.053 & 0.059 & 0.200 \\
\hline 0.299 & 0.366 & 0.049 & 0.053 & 0.150 \\
\hline $\begin{array}{l}\text { Asphaltene } \\
\text { volume } \\
\text { fraction }\end{array}$ & \multicolumn{5}{|c|}{3} & $\mathrm{R}_{1}$ (upper phase \\
\hline
\end{tabular}




\begin{tabular}{|l|l|l|l|l|}
\hline 0.053 & 0.070 & 0.114 & 0.133 & 0.870 \\
\hline 0.156 & 0.198 & 0.105 & 0.119 & 0.590 \\
\hline 0.167 & 0.211 & 0.104 & 0.117 & 0.557 \\
\hline 0.179 & 0.226 & 0.102 & 0.114 & 0.520 \\
\hline 0.218 & 0.271 & 0.098 & 0.109 & 0.440 \\
\hline 0.280 & 0.342 & 0.090 & 0.098 & 0.280 \\
\hline 0.300 & 0.364 & 0.088 & 0.095 & 0.180 \\
\hline 0.190 & 0.239 & 0.101 & 0.113 & 0.500 \\
\hline 0.317 & 0.383 & 0.085 & 0.091 & 0.130 \\
\hline
\end{tabular}

\begin{tabular}{|c|c|c|c|c|}
\hline \multicolumn{5}{|c|}{4} \\
\hline $\begin{array}{l}\text { Asphaltene } \\
\text { volume } \\
\text { fraction } \\
\end{array}$ & $\begin{array}{c}\text { Asphaltene mass } \\
\text { fraction }\end{array}$ & $\begin{array}{l}\text { Polymer volume } \\
\text { fraction }\end{array}$ & $\begin{array}{l}\text { Polymer mass } \\
\text { fraction }\end{array}$ & $\begin{array}{l}\mathrm{R}_{1} \text { (upper phase } \\
\text { volume fraction) }\end{array}$ \\
\hline 0.125 & 0.158 & 0.175 & 0.197 & 0.840 \\
\hline 0.158 & 0.198 & 0.168 & 0.187 & 0.690 \\
\hline 0.196 & 0.243 & 0.161 & 0.177 & 0.580 \\
\hline 0.270 & 0.327 & 0.146 & 0.157 & 0.380 \\
\hline 0.230 & 0.282 & 0.154 & 0.168 & 0.500 \\
\hline 0.280 & 0.338 & 0.144 & 0.155 & 0.350 \\
\hline 0.290 & 0.349 & 0.142 & 0.152 & 0.310 \\
\hline 0.310 & 0.371 & 0.138 & 0.147 & 0.240 \\
\hline 0.330 & 0.393 & 0.134 & 0.142 & 0.180 \\
\hline
\end{tabular}

\begin{tabular}{|c|c|c|c|c|}
\hline \multicolumn{5}{|c|}{5} \\
\hline $\begin{array}{c}\text { Asphaltene } \\
\text { volume } \\
\text { fraction }\end{array}$ & $\begin{array}{c}\text { Asphaltene mass } \\
\text { fraction }\end{array}$ & $\begin{array}{c}\text { Polymer volume } \\
\text { fraction }\end{array}$ & $\begin{array}{c}\text { Polymer mass } \\
\text { fraction }\end{array}$ & $\begin{array}{c}\mathrm{R}_{1} \text { (upper phase } \\
\text { volume fraction) }\end{array}$ \\
\hline 0.160 & 0.197 & 0.240 & 0.263 & 0.850 \\
\hline 0.200 & 0.244 & 0.231 & 0.251 & 0.700 \\
\hline 0.244 & 0.294 & 0.220 & 0.236 & 0.546 \\
\hline 0.260 & 0.312 & 0.215 & 0.229 & 0.510 \\
\hline 0.330 & 0.388 & 0.200 & 0.209 & 0.320 \\
\hline
\end{tabular}




\begin{tabular}{|l|l|l|l|l|}
\hline 0.352 & 0.412 & 0.190 & 0.198 & 0.230 \\
\hline 0.370 & 0.431 & 0.185 & 0.192 & 0.160 \\
\hline
\end{tabular}

\begin{tabular}{|c|c|c|c|c|}
\hline \multicolumn{5}{|c|}{6} \\
$\begin{array}{c}\text { Asphaltene } \\
\text { volume } \\
\text { fraction }\end{array}$ & $\begin{array}{c}\text { Asphaltene mass } \\
\text { fraction }\end{array}$ & $\begin{array}{c}\text { Polymer volume } \\
\text { fraction }\end{array}$ & $\begin{array}{c}\text { Polymer mass } \\
\text { fraction }\end{array}$ & $\begin{array}{c}\mathrm{R}_{1} \text { (upper phase } \\
\text { volume fraction) }\end{array}$ \\
\hline 0.300 & 0.350 & 0.291 & 0.302 & 0.460 \\
\hline 0.280 & 0.330 & 0.272 & 0.285 & 0.480 \\
\hline 0.250 & 0.299 & 0.243 & 0.259 & 0.540 \\
\hline 0.240 & 0.289 & 0.233 & 0.249 & 0.570 \\
\hline 0.230 & 0.278 & 0.223 & 0.240 & 0.590 \\
\hline
\end{tabular}

\begin{tabular}{|c|c|c|c|c|}
\hline \multicolumn{5}{|c|}{7} \\
\hline $\begin{array}{c}\text { Asphaltene } \\
\text { volume } \\
\text { fraction }\end{array}$ & $\begin{array}{c}\text { Asphaltene mass } \\
\text { fraction }\end{array}$ & $\begin{array}{c}\text { Polymer volume } \\
\text { fraction }\end{array}$ & $\begin{array}{c}\text { Polymer mass } \\
\text { fraction }\end{array}$ & $\begin{array}{c}\mathrm{R}_{1} \text { (upper phase } \\
\text { volume fraction) }\end{array}$ \\
\hline 0.41 & 0.422 & 0.296 & 0.300 & 0.310 \\
\hline 0.39 & 0.403 & 0.280 & 0.287 & 0.350 \\
\hline
\end{tabular}


Table S.11: Compositions and associated polymer-rich phase volume data for pentane asphaltene + polystyrene + toluene (based on X-ray measurements) for trajectories $p, q$, and $\mathrm{r}$.

\begin{tabular}{|c|c|c|c|c|c|}
\hline \multirow{7}{*}{$\mathrm{p}$} & $\begin{array}{l}\text { Asphaltene } \\
\text { volume } \\
\text { fraction }\end{array}$ & $\begin{array}{l}\text { Asphaltene } \\
\text { mass } \\
\text { fraction }\end{array}$ & $\begin{array}{l}\text { Polystyrene } \\
\text { volume } \\
\text { fraction }\end{array}$ & $\begin{array}{l}\text { Polystyrene } \\
\text { mass } \\
\text { fraction }\end{array}$ & $\begin{array}{l}\text { Volume fraction of } \\
\text { the upper phase (R) }\end{array}$ \\
\hline & 0.065 & 0.086 & 0.042 & 0.050 & 0.754 \\
\hline & 0.059 & 0.079 & 0.039 & 0.046 & 0.797 \\
\hline & 0.055 & 0.073 & 0.035 & 0.042 & 0.850 \\
\hline & 0.050 & 0.067 & 0.033 & 0.039 & 0.896 \\
\hline & 0.047 & 0.063 & 0.030 & 0.036 & 0.930 \\
\hline & 0.044 & 0.059 & 0.028 & 0.033 & 0.956 \\
\hline \multirow{6}{*}{ q } & 0.103 & 0.135 & 0.030 & 0.035 & 0.464 \\
\hline & 0.094 & 0.124 & 0.028 & 0.033 & 0.505 \\
\hline & 0.086 & 0.114 & 0.025 & 0.029 & 0.553 \\
\hline & 0.079 & 0.105 & 0.023 & 0.027 & 0.615 \\
\hline & 0.074 & 0.098 & 0.022 & 0.026 & 0.650 \\
\hline & 0.069 & 0.092 & 0.020 & 0.024 & 0.689 \\
\hline \multirow{6}{*}{$\mathrm{r}$} & 0.130 & 0.169 & 0.024 & 0.028 & 0.282 \\
\hline & 0.119 & 0.156 & 0.022 & 0.026 & 0.313 \\
\hline & 0.110 & 0.144 & 0.020 & 0.024 & 0.346 \\
\hline & 0.100 & 0.132 & 0.018 & 0.022 & 0.370 \\
\hline & 0.094 & 0.124 & 0.017 & 0.020 & 0.393 \\
\hline & 0.088 & 0.117 & 0.016 & 0.019 & 0.422 \\
\hline
\end{tabular}


Table S 12: Compositions and associated polymer-rich phase volume data for retentate + polystyrene + toluene (based on X-Ray measurements) for trajectories $1,2,3,4,5,6,7$

\begin{tabular}{|c|c|c|c|c|}
\hline $\begin{array}{c}\text { Asphaltene } \\
\text { volume } \\
\text { fraction }\end{array}$ & $\begin{array}{c}\text { Asphaltene mass } \\
\text { fraction }\end{array}$ & $\begin{array}{c}\text { polymer volume } \\
\text { fraction }\end{array}$ & $\begin{array}{c}\text { Polymer mass } \\
\text { fraction }\end{array}$ & $\begin{array}{c}\mathrm{R}_{1} \text { (upper phase } \\
\text { volume fraction) }\end{array}$ \\
\hline 0.030 & 0.040 & 0.044 & 0.044 & 0.850 \\
\hline 0.048 & 0.063 & 0.043 & 0.043 & 0.670 \\
\hline 0.070 & 0.091 & 0.042 & 0.042 & 0.530 \\
\hline 0.080 & 0.104 & 0.041 & 0.041 & 0.470 \\
\hline 0.112 & 0.143 & 0.040 & 0.04 & 0.390 \\
\hline 0.140 & 0.176 & 0.039 & 0.039 & 0.330 \\
\hline 0.172 & 0.212 & 0.037 & 0.037 & 0.290 \\
\hline 0.211 & 0.255 & 0.036 & 0.036 & 0.160 \\
\hline
\end{tabular}

\begin{tabular}{|c|c|c|c|c|}
\hline \multicolumn{5}{|c|}{2} \\
$\begin{array}{c}\text { Asphaltene } \\
\text { volume } \\
\text { fraction }\end{array}$ & $\begin{array}{c}\text { Asphaltene mass } \\
\text { fraction }\end{array}$ & $\begin{array}{c}\text { Polymer volume } \\
\text { fraction }\end{array}$ & $\begin{array}{c}\text { Polymer mass } \\
\text { fraction }\end{array}$ & $\begin{array}{c}\mathrm{R}_{1} \text { (upper phase } \\
\text { volume fraction) }\end{array}$ \\
\hline 0.037 & 0.049 & 0.091 & 0.091 & 0.850 \\
\hline 0.070 & 0.090 & 0.088 & 0.088 & 0.680 \\
\hline 0.100 & 0.127 & 0.085 & 0.085 & 0.570 \\
\hline 0.120 & 0.151 & 0.083 & 0.083 & 0.500 \\
\hline 0.190 & 0.230 & 0.076 & 0.076 & 0.280 \\
\hline 0.211 & 0.253 & 0.075 & 0.075 & 0.200 \\
\hline 0.240 & 0.284 & 0.071 & 0.071 & 0.130 \\
\hline
\end{tabular}




\begin{tabular}{|c|c|c|c|c|}
\hline \multicolumn{5}{|c|}{3} \\
\hline $\begin{array}{c}\text { Asphaltene } \\
\text { volume } \\
\text { fraction }\end{array}$ & $\begin{array}{c}\text { Asphaltene mass } \\
\text { fraction }\end{array}$ & $\begin{array}{c}\text { Polymer volume } \\
\text { fraction }\end{array}$ & $\begin{array}{c}\text { Polymer mass } \\
\text { fraction }\end{array}$ & $\begin{array}{c}\mathrm{R}_{1} \text { (upper phase } \\
\text { volume fraction) }\end{array}$ \\
\hline 0.050 & 0.065 & 0.138 & 0.138 & 0.880 \\
\hline 0.080 & 0.102 & 0.133 & 0.133 & 0.730 \\
\hline 0.120 & 0.150 & 0.127 & 0.127 & 0.580 \\
\hline 0.160 & 0.195 & 0.121 & 0.121 & 0.460 \\
\hline 0.200 & 0.239 & 0.115 & 0.115 & 0.320 \\
\hline 0.240 & 0.282 & 0.109 & 0.109 & 0.200 \\
\hline 0.260 & 0.302 & 0.106 & 0.106 & 0.140 \\
\hline
\end{tabular}

\begin{tabular}{|c|c|c|c|c|}
\hline \multicolumn{1}{|c|}{4} \\
$\begin{array}{c}\text { Asphaltene } \\
\text { volume } \\
\text { fraction }\end{array}$ & $\begin{array}{c}\text { Asphaltene mass } \\
\text { fraction }\end{array}$ & $\begin{array}{c}\text { Polymer volume } \\
\text { fraction }\end{array}$ & $\begin{array}{c}\text { Polymer mass } \\
\text { fraction }\end{array}$ & $\begin{array}{c}\mathrm{R}_{1} \text { (upper phase } \\
\text { volume fraction) }\end{array}$ \\
\hline 0.100 & 0.124 & 0.188 & 0.188 & 0.920 \\
\hline 0.120 & 0.148 & 0.184 & 0.184 & 0.810 \\
\hline 0.150 & 0.182 & 0.178 & 0.178 & 0.670 \\
\hline 0.190 & 0.226 & 0.169 & 0.169 & 0.460 \\
\hline 0.220 & 0.258 & 0.163 & 0.163 & 0.320 \\
\hline 0.250 & 0.289 & 0.158 & 0.158 & 0.160 \\
\hline 0.265 & 0.304 & 0.153 & 0.153 & 0.100 \\
\hline
\end{tabular}

\begin{tabular}{|c|c|c|c|c|}
\hline \multicolumn{5}{|c|}{5} \\
\hline $\begin{array}{c}\text { Asphaltene } \\
\text { volume } \\
\text { fraction }\end{array}$ & $\begin{array}{c}\text { Asphaltene mass } \\
\text { fraction }\end{array}$ & $\begin{array}{c}\text { Polymer volume } \\
\text { fraction }\end{array}$ & $\begin{array}{c}\text { Polymer mass } \\
\text { fraction }\end{array}$ & $\begin{array}{c}\mathrm{R}_{1} \text { (upper phase } \\
\text { volume fraction) }\end{array}$ \\
\hline 0.230 & 0.267 & 0.182 & 0.182 & 0.310 \\
\hline 0.220 & 0.258 & 0.174 & 0.174 & 0.290 \\
\hline
\end{tabular}




\begin{tabular}{|c|c|c|c|c|}
\hline \multicolumn{5}{|c|}{6} \\
\hline $\begin{array}{c}\text { Asphaltene } \\
\text { volume } \\
\text { fraction }\end{array}$ & $\begin{array}{c}\text { Asphaltene mass } \\
\text { fraction }\end{array}$ & $\begin{array}{c}\text { Polymer volume } \\
\text { fraction }\end{array}$ & $\begin{array}{c}\text { Polymer mass } \\
\text { fraction }\end{array}$ & $\begin{array}{c}\mathrm{R}_{1} \text { (upper phase } \\
\text { volume fraction) }\end{array}$ \\
\hline 0.210 & 0.245 & 0.215 & 0.215 & 0.470 \\
\hline 0.195 & 0.230 & 0.199 & 0.199 & 0.440 \\
\hline
\end{tabular}

\begin{tabular}{|c|c|c|c|c|}
\hline \multicolumn{5}{|c|}{7} \\
\hline $\begin{array}{c}\text { Asphaltene } \\
\text { volume } \\
\text { fraction }\end{array}$ & $\begin{array}{c}\text { Asphaltene mass } \\
\text { fraction }\end{array}$ & $\begin{array}{c}\text { Polymer volume } \\
\text { fraction }\end{array}$ & $\begin{array}{c}\text { Polymer mass } \\
\text { fraction }\end{array}$ & $\begin{array}{c}\mathrm{R}_{1} \text { (upper phase } \\
\text { volume fraction) }\end{array}$ \\
\hline 0.115 & 0.142 & 0.198 & 0.198 & 0.800 \\
\hline 0.105 & 0.130 & 0.181 & 0.181 & 0.840 \\
\hline
\end{tabular}

Table S.13: Computed binodal points for pentane asphaltene + polystyrene + toluene based on X-ray measurements for trajectories $\mathrm{p}, \mathrm{q}, \mathrm{r}$ in Table S.11 and the method of Bondar et al. ${ }^{40}$

\begin{tabular}{|c|c|}
\hline Asphaltene volume fraction & Polystyrene volume fraction \\
\hline 0.034 & 0.058 \\
\hline 0.035 & 0.066 \\
\hline 0.036 & 0.050 \\
\hline 0.036 & 0.042 \\
\hline 0.038 & 0.035 \\
\hline 0.039 & 0.028 \\
\hline 0.138 & 0.013 \\
\hline 0.144 & 0.012 \\
\hline 0.153 & 0.011 \\
\hline 0.158 & 0.010 \\
\hline 0.168 & 0.010 \\
\hline 0.176 & 0.010 \\
\hline
\end{tabular}

